\title{
BMJ Open Cost-effectiveness analysis of PET/CT surveillance imaging to detect systemic recurrence in resected stage III melanoma: study protocol
}

\author{
Mbathio Dieng, ${ }^{1}$ Nikita Khanna (D , ${ }^{1}$ Mai Thi Hoang Nguyen, ${ }^{1}$ Robin Turner, ${ }^{2}$ \\ Sarah J Lord, ${ }^{1}$ Alexander M Menzies, ${ }^{3,4}$ Jay Allen, ${ }^{3}$ Robyn Saw, ${ }^{3}$ Omgo E Nieweg, ${ }^{3,5}$ \\ John Thompson, ${ }^{6,7}$ Rachael L Morton (i) ${ }^{1}$
}

To cite: Dieng M, Khanna N, Nguyen MTH, et al. Costeffectiveness analysis of PET/ CT surveillance imaging to detect systemic recurrence in resected stage III melanoma: study protocol. BMJ Open 2020;10:e037857. doi:10.1136/ bmjopen-2020-037857

- Prepublication history for this paper is available online. To view these files, please visit the journal online (http://dx.doi org/10.1136/bmjopen-2020037857).

Received 19 February 2020 Revised 29 June 2020 Accepted 11 August 2020

Check for updates

(C) Author(s) (or their employer(s)) 2020. Re-use permitted under CC BY-NC. No commercial re-use. See rights and permissions. Published by BMJ.

For numbered affiliations see end of article.

Correspondence to

Mbathio Dieng;

mbathio.dieng@sydney.edu.au

\section{ABSTRACT}

Introduction In the new era of effective systemic therapies for advanced melanoma, early detection of lower volume recurrent disease using surveillance imaging can improve survival. However, intensive imaging follow-up strategies are likely to increase costs to health systems and may pose risks to patients. The objective of this study is to estimate from the Australian health system perspective the cost-effectiveness of four follow-up strategies in resected stage III melanoma over a 5-year period following surgical treatment with curative intent. Methods and analysis A decision-analytic model will be built to estimate the costs and benefits of (1) 12 monthly, (2) 6 monthly, (3) 3-4 monthly positron emission tomography/CT imaging for 5 years, compared with (4) no imaging follow-up. The model will be populated with probabilities of disease recurrence, test performance measures using data from $>1000$ consecutive resected stage III melanoma patients from Melanoma Institute Australia diagnosed between 2000 and 2017. Healthcare resource use, including surveillance imaging, doctor's visits, subsequent tests and procedures to investigate suspicious findings, will be quantified from detailed patient records and valued using Australian reference pricing. Economic outcomes include cost per new distant melanoma recurrence detected and cost per diagnostic error avoided, for no imaging compared with the other strategies.

Deterministic sensitivity analyses will examine the robustness of model results.

Ethics and dissemination This study was approved by the Sydney Local Health District, Sydney Local Health District Ethics Review Committee (RPAH Zone), $\mathrm{AU} / 1 / 830638$ and the Australian Institute of Health and Welfare (E02019-1-454). The results of this study will be published in peer-reviewed medical and health economics journals and will inform melanoma management guidelines.

\section{INTRODUCTION}

\section{Melanoma}

In Australia, melanoma is the third most common cancer in both men and women, accounting for $10 \%$ of all new cases (13 280:
Strengths and limitations of this study

- The analysis will be based on test accuracy and prevalence data from a cohort study of over 1000 patients with stage III melanoma.

- The model structure accounts for clinical and policyrelevant outcomes including test accuracy.

- The comparison of the three imaging schedules was not based on data from randomised trials and some selection bias might be introduced.

- Treatment cost data will be obtained from the published literature.

7850 women and 5440 men) and $3.8 \%$ of cancer deaths (1775: 545 women and 1230 men) in 2016. ${ }^{1}$ Its incidence is continuing to rise slowly in both sexes, and while mortality and 5-year survival (95\%) after melanoma diagnosis are stable, the disease remains a leading cause of cancer death in young adults aged under 40 .

Stage III melanoma is defined as the presence of metastatic disease in regional lymph nodes and/or the presence of intransit/satellite/microsatellite metastasis. ${ }^{2} 3$ The dissemination of melanoma to regional nodes and visceral organs, such as the lung, liver or brain, implies a poor prognosis. The risk of recurrence is highest in the first 3 years after diagnosis, ${ }^{4}$ but patients have a life long risk of relapse. Appropriate surveillance can identify disease recurrence and enable early individualised treatment. ${ }^{5}$ However, there is no randomised trial evidence that early recognition and treatment of systemic recurrence improve survival outcomes. ${ }^{3}$

\section{Follow-up strategies}

In melanoma clinics, after treatment for initial stage III disease, patients are routinely followed up for at least 5 years ${ }^{36}$ with clinical 
examination and various imaging tests. CT, positron emission tomography (PET) or PET/CT specifically assess for evidence of systemic recurrence. CT is a medical imaging modality that uses ionising radiation and computer processing to generate detailed anatomical scans of the body. Whole-body CT including chest, abdomen and pelvis \pm the brain can detect cancer metastases as small as 2-4 mm. ${ }^{7}$ Reported CT detection rates range from as high as $72 \%$ of distant metastases in asymptomatic patients in one study using clinical follow-up as reference standard ${ }^{8}$ to $15 \%-28 \%$ in another study with whole-body MRI as reference standard. ${ }^{9}$ Drawbacks to CT are its limited soft tissue contrast, cost and radiation exposure, with an effective dose of up to $20 \mathrm{mSv}$ per test. ${ }^{10}$

PET registers metabolic and biochemical activity, however, the images produced lack fine anatomical detail. Images from both modalities (CT and PET) can be taken sequentially, combined and superimposed to create one functional and detailed imaging sequence. This PET/ CT combines functional imaging from the PET scan with specific anatomical images from the CT; if any areas of tumour activity are detected, the superimposed CT scan pinpoints the location. High accuracy of PET/CT has been reported by recent studies assessing the ability of $\mathrm{PET} / \mathrm{CT}$ to detect distant metastases. Danielsen et a $\tilde{\varphi}$ pooled the findings for seven studies that investigated PET/CT in follow-up of cutaneous malignant melanoma and found a pooled sensitivity and negative predictive value of $96 \%$ and $95 \%$. Another systematic review by Rodriguez $e t a l^{11}$ reported an overall sensitivity of $89.4 \%$ and a specificity of $88.8 \%$ in detecting metastases in stage III melanoma patients.

Surveillance imaging during follow-up for resected stage III melanoma patients at risk of systemic recurrence remains controversial. ${ }^{12}{ }^{13}$ Potential benefits include early detection of distant disease that induces a change in management (eg, treatment with surgery, systemic therapies, radiotherapy or enrolment in clinical trials of new treatments), which may lead to better survival outcomes than if patients were diagnosed and treated once symptomatic (with higher disease burden). ${ }^{14}$ Patients also report feeling reassured by regular imaging. ${ }^{15}$ The disadvantages include exposure to radiation that can increase the risk of cancer in the future ${ }^{1617}$; incidental findings that are later found to be harmless, causing unnecessary investigations and anxiety ${ }^{18}$; added downstream tests including both image-guided biopsies and short-term additional cross-sectional imaging follow-up as a result of equivocal findings, to prove or rule out melanoma metas$\operatorname{tasis}^{19}$ and possible complications of these tests, such as bleeding from biopsy of a suspected visceral metastasis. However, not all incidental findings will later be found to be harmless. Some will be treated and the patient will get better, and it is only by looking at population-level mortality data that we are able to ascertain that some of these cancers must have harmless.

\section{Current guidelines}

International guidelines for follow-up after treatment of stage III melanoma vary considerably with respect to surveillance imaging, illustrating the paucity of high-level evidence. The German guidelines recommend CT or PET/CT every 6 months for the first 3 years for resected stage III patients ${ }^{20}$; The US guidelines recommend imaging every 4-12 months for the first 5 years, ${ }^{21}$ while the UK National Institute for Health and Care Excellence guidelines recommend surveillance imaging only if there is a clinical trial investigating the value of regular imaging or a local policy with specific funding for 6 monthly imaging for 3 years. ${ }^{22}$ The Australian Melanoma Guidelines (2018) state CT of the chest, abdomen and pelvis or PET may be performed prior to definitive therapy where the detection of metastatic disease would influence management. ${ }^{3}$ Ultrasound assessment can detect locoregional recurrence, but it is not applicable for detection of systemic recurrence, and not recommended by Australian guidelines. Currently there is no high-level evidence that early detection and management of recurrence from surveillance imaging improves patient outcomes.

\section{Cost-effectiveness of surveillance imaging}

Few research studies have investigated the costeffectiveness of a surveillance imaging strategy in resected stage III melanoma patients. ${ }^{23-25}$ Mostly the published studies have reported costs and benefits of one-off (baseline) imaging prior to surgery or imaging to assess treatment response in the management of distant (stage IV) disease as well as exploring whether annual imaging to detect systemic recurrence for resected stage III melanoma patients is more effective than no annual imaging, there is a need to investigate and establish its relative cost-effectiveness for the identification of melanoma recurrence.

The follow-up strategies in current practice were developed before potentially effective systemic therapies that were available to treat advanced melanoma and also before patients with stage III melanoma received adjuvant therapy (where different adjuvant treatments have different recurrence risks, particularly in the first 1-2 years). These new therapies have significant benefits for patients, and in the metastatic setting, the potential to be most efficacious in patients with lower disease burden. ${ }^{26}$ Therefore, it might be beneficial to identify recurrence earlier for patients who might benefit more from earlier systemic treatment. This study aims to investigate the 'opportunity cost' of surveillance imaging by exploring the cost-effectiveness of four surveillance strategies (see table 1) for the diagnosis and treatment of distant melanoma recurrence by modelling the relative costs and benefits accrued from (a) no- scheduled imaging follow-up compared with: (b) 12 monthly imaging: one PET/CT scan per year for 5 years, (c) 6monthly imaging: two PET/CT scans per year for 5 years, (d) 3-4 monthly $\mathrm{PET} / \mathrm{CT}$ imaging for 5 years: (involves routine imaging 
Table 1 Follow-up strategy description

\begin{tabular}{|c|c|c|}
\hline & Follow-up strategy & Description \\
\hline Intervention & No imaging follow-up & $\begin{array}{l}\text { No further routine imaging during follow-up. } \\
\text { Clinical visit every } 4 \text { months for the first } 3 \text { years, every } 6 \text { months in years } \\
4-5 \text {. Patients receive imaging if either the patient or doctor identifies signs/ } \\
\text { symptoms suggesting recurrence }\end{array}$ \\
\hline \multirow[t]{2}{*}{ Comparators } & $\begin{array}{l}\text { Intensive surveillance } \\
\text { imaging }\end{array}$ & $\begin{array}{l}\text { Patients given routine imaging every } 3-4 \text { months during the first } 3 \text { years, } \\
\text { every } 6 \text { months in years } 4-5 \text {. Clinical visit with a melanoma specialist at the } \\
\text { time of each scan }\end{array}$ \\
\hline & $\begin{array}{l}\text { Routine 12-monthly } \\
\text { imaging }\end{array}$ & $\begin{array}{l}\text { One PET/CT scan per year for } 5 \text { years. } \\
\text { Clinical visit with a melanoma specialist at the time of the scan }\end{array}$ \\
\hline
\end{tabular}

PET, positron emission tomography.

every 3 months during the first 3 years, every 6 months in years 4-5).

\section{METHODS AND ANALYSIS \\ Patients and data source}

This prospective cohort study identified consecutive resected stage III melanoma patients with American Joint Cancer Committee eighth edition stage III disease, ${ }^{2}$ treated at Melanoma Institute Australia (MIA) between the years 2000 and 2017 and entered into the research database at MIA. We included stage IIIA-D melanoma patients who had received surgical treatment with curative intent had no evidence of further disease postoperatively and were followed-up clinically at MIA to assess for recurrence. Many patients underwent repeated surveillance imaging as a part of their scheduled follow-up. We also identified a cohort of patients with standard clinical follow-up that did not include surveillance imaging.

\section{Patientand public involvement}

No patient involved.

\section{Model structure}

To assess the cost-effectiveness of a no-imaging follow-up strategy compared with 12 monthly, 6 monthly and 3-4 monthly imaging, a decision tree will be built in TreeAge software (TreeAge Software, Williamstown, Massachusetts, USA). The model will start with patients receiving one of four follow-up strategies: (1) routine 12 monthly imaging, (2) routine 6 monthly imaging, (3) 3-4 monthly imaging or (4) follow-up without imaging (see figure 1). Each branch of the decision tree will represent a follow-up strategy under assessment and will be parameterised with data on diagnostic accuracy, costs and recurrence obtained from our prospective cohort and from published literature over a time horizon of 5 years. Two economic outcomes will be assessed: cost per case of distant recurrence appropriately identified and cost per diagnosis error avoided.

\section{Model assumption}

The model assumptions include:

a. Surveillance imaging is primarily used to identify systemic recurrence. Locoregional melanoma recurrence is often identified by physical examination in contrast to distant disease, which is difficult to detect by physical examinations. Thus PET/CT imaging surveillance is predominantly undertaken to identify distant metastatic disease. ${ }^{27}$

b. Systemic recurrence occurs only once for patients who progress. Therefore, the model will simulate the costs and benefits up to the first (initial) systemic recurrence.

c. In the absence of histopathology, disease status after 6 months of clinical follow-up will be used as a reference standard for PET/CT imaging.

d. Based on Australian guidelines, ${ }^{3}$ patients with positive imaging test results will undergo confirmatory investigations: fine-needle aspiration biopsy, core biopsy, serum lactate dehydrogenase, whole body PET/CT, MRI of the brain and gene mutation testing.

e. The costs for unresectable stage III or stage IV melanoma treatments included standard imaging, medical appointments, genetic testing and pharmacotherapies where relevant as well as costs for grades 3 and 4 adverse events for pharmacotherapies (eg, colitis).

f. Costs of palliative and end of life care will be excluded. As the main outcomes, the effectiveness end point applied was the detection and treatment of distant recurrence, the costs of end of life care were not included in the economic analysis.

\section{IMAGING SURVEILLANCE STRATEGIES}

We will evaluate the ability of the four surveillance strategies to detect systemic recurrence for each patient in the respective cohort. Table 1 describes the different follow-up strategies. 


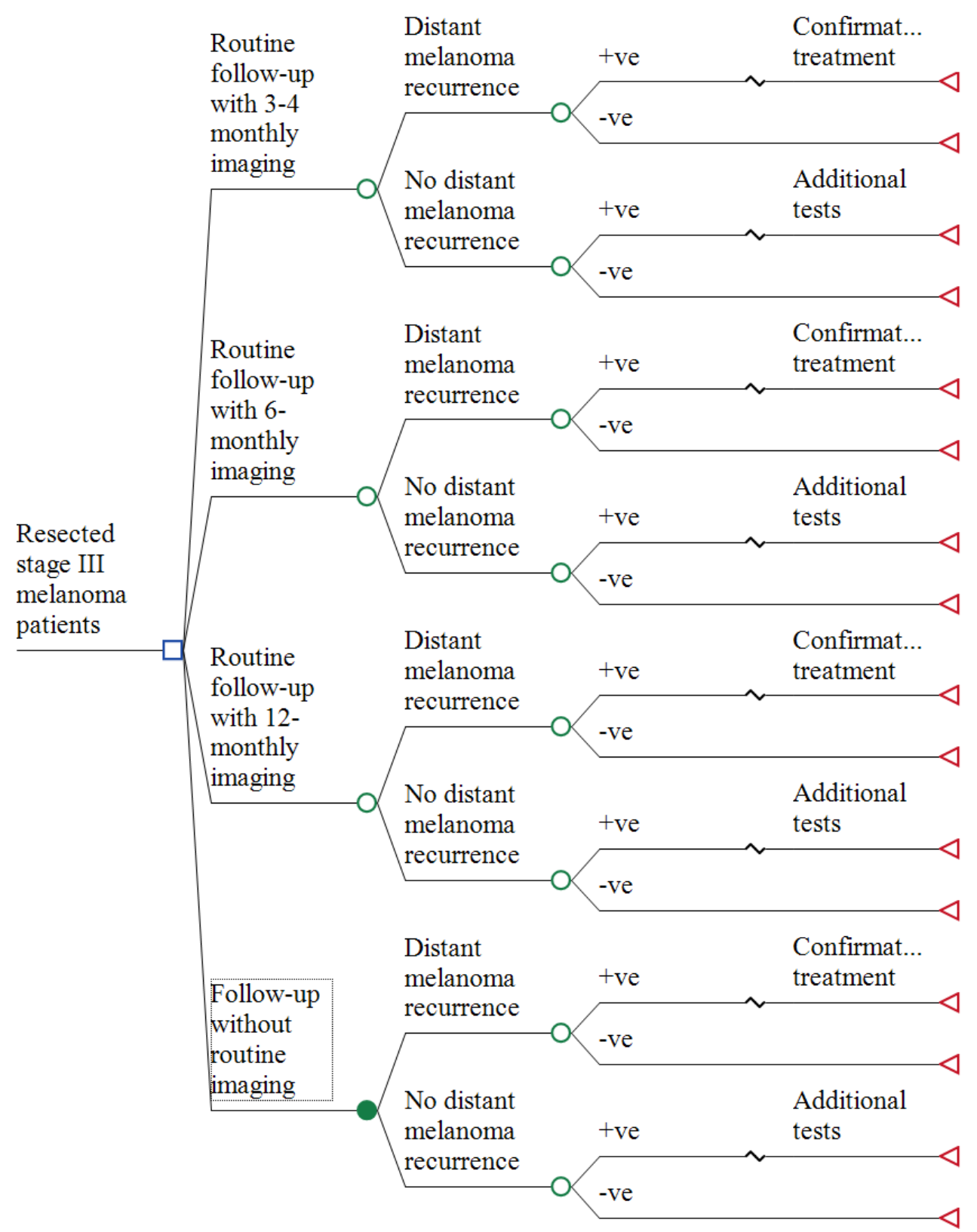

Figure 1 Decision model structure. PET, positron emission tomography.

\section{DATA REQUIRED FOR THE MODEL \\ Prevalence data}

We will populate the decision model with the prevalence of distant melanoma recurrence from our MIA cohort. For each follow-up strategy, the prevalence of systemic recurrence will be calculated.

\section{Test accuracy data}

$\mathrm{PET} / \mathrm{CT}$ sensitivity and specificity over a 5-year period will be derived from a cohort study we conducted among 332 resected stage III melanoma patients (median follow-up 61 months). This analysis has provided data regarding the diagnostic accuracy of PET/CT at each 3, 6 and 12 monthly follow-up time points. (table 2). The result of PET/CT imaging will be classified as true positive (TP), if metastatic disease was detected by the surveillance imaging. PET/CT findings will be defined as true negative $(\mathrm{TN})$, if the scan was negative and no distant disease was detected during further follow-up. $\mathrm{PET} / \mathrm{CT}$ results will be defined as false negative (FN), if the scan was negative, but recurrent disease was detected during 6-month follow-up by other tests or physical examination in clinical follow-up. PET/CT findings will be defined as false positive (FP), if the scan indicated melanoma or suspicion for melanoma, but the reference standard confirmed there was no melanoma.

\section{Healthcare resource use and costs}

Healthcare resources will include all imaging, confirmatory tests and blood tests, medical appointments and subsequent treatment, following the initial surgical management of stage III melanoma (table 3). Healthcare use incurred to diagnose stage III disease or to treat it surgically will be excluded. To account for the cost of 
Table 2 Test performance of PET/CT surveillance imaging

\begin{tabular}{llll}
\hline Investigation & Sensitivity (95\% Cl) & Specificity (95\% Cl) & Reference \\
\hline 3-4 monthly imaging & $79 \%(70 \%$ to $86 \%)$ & $88.6 \%(86.4$ to 90.5$)$ & 32 \\
12-monthly imaging & $79 \%(70 \%$ to $86 \%)$ & $88.6 \%(86.4$ to 90.5$)$ & 32 \\
6-monthly imaging & $79 \%(70 \%$ to $86 \%)$ & $88.6 \%(86.4$ to 90.5$)$ & 32 \\
No imaging follow-up & $71.4 \%(55.4-84.3)$ & $99.6 \%(99.2$ to 99.8$)$ & 33 \\
\hline
\end{tabular}

$\mathrm{PET}$, positron emission tomography.

incidental findings, our model will include the costs of further investigations (eg, extra scans, diagnostic biopsies) following an FP result resource use will be identified from each patient's detailed medical records at MIA.

The costs of resource utilisation will be estimated from the perspective of the Australian health system. Resource use will be valued using unit costs from the Medicare Benefits Schedule for diagnostic tests and outpatient care and Australian Refined Diagnosis Related Groups for hospital admissions. ${ }^{28}$ Treatment costs following a melanoma recurrence or stage IV diagnosis will be derived from published Australian study. ${ }^{29}$ All costs will be adjusted to 2020 prices and $5 \%$ discounting per annum will be applied. ${ }^{30}$

\section{Outcomes}

We will report two different effectiveness/outcome measures from an Australian health system perspective: the cost per new distant melanoma recurrence detected and treated, and the cost per diagnostic error avoided.

\section{Outcome for recurrent melanoma appropriately diagnosed}

For distant recurrent melanoma appropriately diagnosed and treated, we will use the difference between the TP and $\mathrm{FN}$ value from our cohort study. We will give a value of 1 to TP and 0 to FN. For convenience, we will give the value 1 for $\mathrm{FP}$ and $\mathrm{TN}$, therefore, we will only give the value of 0 for an erroneous identification.

Outcome for diagnostic error avoided

For the outcome of diagnostic error avoided, no effectiveness data are required. The $\mathrm{TP}$ and $\mathrm{TN}$ cases will be given the value of 1 . TPs and FNs will be given the value of 0 .

Table 3 Cost inputs for the model, 2019 Australian dollars

\begin{tabular}{|c|c|c|c|c|}
\hline Test & Site & $\begin{array}{l}\text { Base case costs } \\
\text { (AUD) }\end{array}$ & $\begin{array}{l}\text { Sensitivity analysis } \\
\text { range (AUD) }\end{array}$ & Source \\
\hline Whole body PET/CT & Whole body & 1397 & & $\begin{array}{l}\text { MBS \#57007, MBS } \\
\# 61553\end{array}$ \\
\hline Ultrasound & Regional lymph node & 93 & & MBS \#55 812 \\
\hline MRI & Brain and head & 343 & & MBS \#63 001 \\
\hline X-ray & Chest and abdomen & 81 & & $\begin{array}{l}\text { MBS \# 58903, MBS \# } \\
58503\end{array}$ \\
\hline FNAB & $\begin{array}{l}\text { Solid tissue or tissues } \\
\text { from two or more sites }\end{array}$ & 188 & & MBS \# 73066 \\
\hline Bone scan & Whole body & 408 & & MBS \# 61421 \\
\hline Clinical follow-up (Specialist) & & 66 & & MBS \#116 \\
\hline Mutation analysis & $\begin{array}{l}\text { Stage III/IV tumour } \\
\text { tissue }\end{array}$ & 196 & & MBS \# 73336 \\
\hline $\begin{array}{l}\text { Serum lactate dehydrogenase } \\
(\mathrm{LDH})\end{array}$ & & 8 & & MBS \# 66500 \\
\hline $\begin{array}{l}\text { Cost of treatment: } 12-\text { month } \\
\text { cost-stage III unresectable/IV }\end{array}$ & & 115072 & $105208-125573$ & 29 \\
\hline $\begin{array}{l}\text { Cost of treatment: } 36-\text { month } \\
\text { cost-stage III unresectable/IV }\end{array}$ & & 187599 & 175 520-2 00130 & 29 \\
\hline
\end{tabular}

AUD, Australian dollar; FNAB, fine needle aspiration biopsy. 


\section{Analysis}

The model will estimate the mean cost for each follow-up strategy and patients entering the model will be aged 18 years or older. The results of the costeffectiveness analysis will be presented in incremental cost-effectiveness ratios (ICERs). The economic outcomes will be the cost per new distant melanoma recurrence appropriately detected and treated and cost per diagnostic error avoided, for the three strategies when compared with no imaging. A deterministic analysis will be carried out for the base-case results for the different outcome measures.

\section{Sensitivity analysis}

A number of sensitivity analyses will be carried out as summarised below:

1. Deterministic sensitivity analyses will be undertaken to explore the effect of differences in test performance and costs of surveillance imaging in terms of the ICERs. A range of sensitivity and specificity values as well as costs will be sourced from the published literature.

2. A deterministic sensitivity analysis will be carried out on the extreme values for the range of prevalence of systemic recurrence found in the published trials and registry studies.

3. Probabilistic sensitivity analysis will be undertaken to determine the uncertainty of the model input parameters of sensitivity and specificity, prevalence and treatment costs. Each model parameter will be assigned a distribution reflecting the amount and pattern of its variation, and cost-effectiveness results will be calculated by simultaneously selecting random values from each distribution. The process will be repeated 10000 times in a Monte Carlo simulation of the model to give an indication of how variation in the model parameters leads to variation in the ICERs for a given imaging strategy. We will place a normal distribution for the data on test accuracy. Treatment costs point estimates will be derived from the literature. ${ }^{29}$ For costs we will place a Gamma distributionand a beta distribution for sensitivity, specificity and prevalence.

Reporting of the study design, methods and results will follow the Consolidated Health Economic Evaluation Reporting Standards. ${ }^{31}$

\section{Ethics and dissemination}

This study was approved by the Institutional Review Board's ethics committee (MIA2016/182), the Australian Institute of Health and Welfare (EO2019-1-454) and the Royal Prince Alfred Hospital Ethics Committee X18-0144, LNR/18/RPAH/206.

The results of this study will inform the Cancer Council Australia's melanoma guidelines Wiki. ${ }^{3}$ Additionally, the results of this study will be published in high-impact peerreviewed medical and economic journals. A lay summary written in collaboration with consumers from Melanoma Patients Australia will be disseminated through social media channels.

\section{DISCUSSION}

After treatment of stage III melanoma, patients have regular follow-up with their treating doctors. Imaging techniques such as CT, PET or PET/CT for detection of distant disease are used alongside these visits. Routine surveillance aims to support and reassure patients and to detect systemic recurrence early so that appropriate treatment can be given in an optimal time frame. Furthermore, with new effective therapies for stage IV disease, robust evidence regarding the most cost-effective approach for follow-up of stage III melanoma patients is needed. There is a lack of consensus and evidence-based guidelines regarding the optimal imaging modalities or the frequency of schedules to best identify asymptomatic systemic melanoma recurrences.

This study will provide a framework for estimating the cost-effectiveness of four follow-up strategies in resected stage III melanoma over a 5-year time frame following curative-intent surgical treatment.

Strengths and limitations: this is one of the first studies to estimate the cost-effectiveness of three follow-up regimens compared with no imaging follow-up in melanoma. Furthermore, the analysis will be based on test accuracy data from a large cohort study of over 1000 patients with stage III and value of Information analysis will estimate the expected value of conducting a prospective randomised controlled trial of imaging surveillance to reduce decision uncertainty. Finally, the model structure accounts for clinical and policy-relevant outcomes including test accuracy.

There are some limitations to this study. First, the comparison of the three imaging schedules was not based on data from randomised trials and some selection bias might be introduced. The model structure was limited in the pathways for which data were unavailable, therefore, we had to make many assumptions. Second, the treatment cost and utility data will be obtained from the published literature. Furthermore, the comparison of the three imaging schedules was not based on data from randomised trials and some selection bias might be introduced.

\section{Author affiliations \\ ${ }^{1}$ NHMRC Clinical Trials Centre, Faculty of Medicine and Health, The University of Sydney, Camperdown, New South Wales, Australia \\ ${ }^{2}$ Biostatistics, University of Otago Dunedin School of Medicine, Dunedin, New Zealand \\ ${ }^{3}$ Melanoma Institute Australia, The University of Sydney, North Sydney, New South Wales, Australia \\ ${ }^{4}$ Department of Medical Oncology, Royal North Shore and Mater Hospitals, Sydney, New South Wales, Australia \\ ${ }^{5}$ Department of Melanoma and Surgical Oncology, Royal Prince Alfred Hospital, Sydney, New South Wales, Australia \\ ${ }^{6}$ Surgical Oncology, Melanoma Institute of Australia, North Sydney, New South Wales, Australia \\ ${ }^{7}$ Sydney Medical School, The University of Sydney, Sydney, New South Wales, Australia}

Twitter Rachael L Morton @RachaelMorton12 
Contributors MD, RLM, RT, SJL, AMM, JA, RS, OEN and JT contributed to the study design; MD, RLM, RT and NK contributed to the statistical analyses; MD, RLM, NK and MTHN contributed to data acquisition; MD, NK and RLM were responsible for the initial drafting of this manuscript; all authors contributed to the review of this manuscript and provided comments. All authors read and approved the final manuscript.

Funding This study was funded by Cancer Australia through the Priority-driven Collaborative Cancer Research Grant Scheme (ID 1129568).

Competing interests RS received honoraria from Novartis and MSD for an advisory capacity.

Patient and public involvement Patients and/or the public were involved in the design, or conduct, or reporting, or dissemination plans of this research. Refer to the Methods section for further details.

Patient consent for publication Not required.

Provenance and peer review Not commissioned; externally peer reviewed.

Open access This is an open access article distributed in accordance with the Creative Commons Attribution Non Commercial (CC BY-NC 4.0) license, which permits others to distribute, remix, adapt, build upon this work non-commercially, and license their derivative works on different terms, provided the original work is properly cited, appropriate credit is given, any changes made indicated, and the use is non-commercial. See: http://creativecommons.org/licenses/by-nc/4.0/.

\section{ORCID iDs}

Nikita Khanna http://orcid.org/0000-0002-4052-3610

Rachael L Morton http://orcid.org/0000-0001-7834-0572

\section{REFERENCES}

1 Australian Institute for Health and Welfare (AlHW). Australian cancer incidence and mortality (ACIM) books: melanoma of the skin, 2015.

2 Gershenwald JE, Scolyer RA, Hess KR, et al. Melanoma staging: evidence-based changes in the American joint Committee on cancer eighth edition cancer staging manual. CA Cancer J Clin 2017:67:472-92.

3 Coit DG, Thompson JA, Algazi A, et al. Melanoma, version 2.2016, NCCN clinical practice guidelines in oncology. J Natl Compr Canc Netw 2016;14:450-73

4 Leon-Ferre RA, Kottschade LA, Block MS, et al. Association between the use of surveillance PET/CT and the detection of potentially salvageable occult recurrences among patients with resected highrisk melanoma. Melanoma Res 2017;27:335-41.

5 Danielsen M, Højgaard L, Kjær A, et al. Positron emission tomography in the follow-up of cutaneous malignant melanoma patients: a systematic review. Am J Nucl Med Mol Imaging 2013;4:17-28.

6 Morton RL FA-B, Dieng M. Surveillance and Follow-Up of Melanoma Patients. In: IB C, ed. Cutaneous melanoma. Switzerland: Springer Nature, 2019.

7 Hausmann D, Kern C, Schröder MT, et al. [Whole-body MRI in preoperative diagnostics of breast cancer--a comparison with [corrected] staging methods according to the S 3 guidelines]. Rofo 2011;183:1130-7.

8 Lacourse E, Baillargeon R, Dupéré V, et al. Two-Year predictive validity of conduct disorder subtypes in early adolescence: a latent class analysis of a Canadian longitudinal sample. J Child Psychol Psychiatry 2010;51:1386-94.

9 Garbe C, Paul A, Kohler-Späth H, et al. Prospective evaluation of a follow-up schedule in cutaneous melanoma patients: recommendations for an effective follow-up strategy. J Clin Oncol 2003;21:520-9.

10 Zager JS, SV KR. Melanoma. New York: Oxford University Press, 2016.
11 Rodriguez Rivera AM, Alabbas H, Ramjaun A, et al. Value of positron emission tomography scan in stage III cutaneous melanoma: a systematic review and meta-analysis. Surg Oncol 2014;23:11-16.

12 Bhutiani N, Egger ME, McMasters KM. Optimizing follow-up assessment of patients with cutaneous melanoma. Ann Surg Oncol 2017;24:861-3.

13 Swetter SM. Commentary: improved patient outcomes remain elusive after intensive imaging surveillance for high-risk melanoma. $J$ Am Acad Dermatol 2016;75:525-7.

14 Staub LP, Lord SJ, Simes RJ, et al. Using patient management as a surrogate for patient health outcomes in diagnostic test evaluation. BMC Med Res Methodol 2012;12:12.

15 Mitchell J, Callaghan P, Street J, et al. The experience of melanoma follow-up care: an online survey of patients in Australia. J Skin Cancer 2014;2014:1-7.

16 Einstein AJ. Beyond the bombs: cancer risks of low-dose medical radiation. Lancet 2012;380:455-7.

17 Fazel R, Krumholz HM, Wang Y, et al. Exposure to low-dose ionizing radiation from medical imaging procedures. N Engl J Med 2009;361:849-57.

18 Booth TC, Jackson A, Wardlaw JM, et al. Incidental findings found in "healthy" volunteers during imaging performed for research: current legal and ethical implications. Br J Radiol 2010;83:456-65.

19 Chen J, Fazel R, Ross JS, et al. Do imaging studies performed in physician offices increase downstream utilization?: an empiric analysis of cardiac stress testing with imaging. JACC CardiovasC Imaging 2011;4:630-7.

20 Pflugfelder A, Kochs C, Blum A, et al. Malignant melanoma S3guideline "diagnosis, therapy and follow-up of melanoma". J Dtsch Dermatol Ges 2013;11 Suppl 6:1-116. 1-26.

21 Network NCC. National comprehensive cancer network: clinical practice guidelines in oncology, melanoma, 2014.

22 NICE. NIfHaCE. Melanoma: assessment and management. NICE guidelines [NG14], 2015.

23 Bastiaannet E, Uyl-de Groot CA, Brouwers AH, et al. CostEffectiveness of adding FDG-PET or CT to the diagnostic work-up of patients with stage III melanoma. Ann Surg 2012;255:771-6.

24 Krug B, Crott R, Roch I, et al. Cost-effectiveness analysis of FDG PET-CT in the management of pulmonary metastases from malignant melanoma. Acta Oncol 2010;49:192-200.

25 Look Hong NJ, Petrella T, Chan K. Cost-effectiveness analysis of staging strategies in patients with regionally metastatic melanoma. $J$ Surg Oncol 2015;111:423-30.

26 Joseph RW, Elassaiss-Schaap J, Kefford R, et al. Baseline tumor size is an independent prognostic factor for overall survival in patients with melanoma treated with pembrolizumab. Clin Cancer Res 2018;24:4960-7.

27 Xing Y, Bronstein Y, Ross Ml, et al. Contemporary diagnostic imaging modalities for the staging and surveillance of melanoma patients: a meta-analysis. J Natl Cancer Inst 2011;103:129-42.

28 Government A. Pricing framework for Australian public hospital services 2019-20. Authority IHP, 2018.

29 Elliott TM, Whiteman DC, Olsen CM, et al. Estimated healthcare costs of melanoma in australia over 3 years post-diagnosis. Appl Health Econ Health Policy 2017;15:805-16.

30 Government A. Overview and rationale of the economic evaluation. Health Do, 2016.

31 Husereau D, Drummond M, Petrou S, et al. Consolidated health economic evaluation reporting standards (cheers) statement. BMJ 2013;346:f1049.

32 Turner RM, Dieng M, Khanna N, et al. Performance of long-term CT and PET/CT surveillance for detection of distant recurrence in patients with resected stage IIIA-D melanoma ( ${ }^{*}$ equal first authors). Ann Surg Oncol 2020. [Epub ahead of print: 6 Oct 2020].

33 Machet L, Nemeth-Normand F, Giraudeau B, et al. Is ultrasound lymph node examination superior to clinical examination in melanoma follow-up? A monocentre cohort study of 373 patients. $\mathrm{Br}$ J Dermatol 2005;152:66-70. 